\title{
A capacidade institucional do Setor Saúde e a resposta à COVID-19 em perspectiva global
}

\author{
The institutional capacity of the Health Sector and the response \\ to COVID-19 in a global perspective
}

Nilson do Rosário Costa (https://orcid.org/0000-0002-8360-4832) ${ }^{1}$

Paulo Roberto Fagundes da Silva (https://orcid.org/0000-0003-0811-4080) ${ }^{1}$

Marcos Junqueira do Lago (https://orcid.org/0000-0002-4776-7298) ${ }^{2}$

Alessandro Jatobá (https://orcid.org/0000-0002-7059-6546) ${ }^{3}$

${ }^{1}$ Departamento de Ciências Sociais, Escola Nacional de Saúde Pública Sergio Arouca, Fundação Oswaldo Cruz (Fiocruz). R. Leopoldo Bulhões 1480, sala 913, Manguinhos. 21041-210 Rio de Janeiro RJ Brasil. nilsondorosario@gmail.com ${ }^{2}$ Faculdade de Ciências Médicas, Universidade Estadual do Rio de Janeiro. Rio de Janeiro RJ Brasil.

${ }^{3}$ Centro de Estudos

Estratégicos, Fiocruz. Rio de Janeiro RJ Brasil.

\begin{abstract}
This study approaches the Global Health Security Index (GHSI) according to the responses to the first cycle of the COVID-19. The GHSI ranks countries' institutional capacity to address biological risks. We analyzed data regarding the spread of COVID-19 pandemic in 50 countries to assess the ability of GHSI to anticipate health risks. The lack of vaccination determined the spread of the COVID-19 in the first cycle of the pandemic in 2020. Country indicators are correlated and demonstrated by descriptive statistics. The clustering method groups countries by similar age composition. The main restriction that can be attributed to the GHSI concerns the preference of biomedical variables for measuring institutional capacity. Our work shows that the pandemic had a significant impact on better-prepared countries, according to the GHSI, to control the spread of diseases and offer more access to health care in 2020. This paper points out that the health sector depended on the cooperation of governments in the adoption of social distancing during the first cycle of the pandemic. The GHSI failed to consider the role of political leaders who challenge severe health risks by vetoing social distancing.
\end{abstract}

Key words Global Health Security Index, COVID-19, Pandemic, Institutional capacity, Comparative analysis
Resumo O artigo analisa o Índice da Segurança Sanitária Global (ISSG) à luz das respostas nacionais ao primeiro ciclo da pandemia da COVID-19. O ISSG classifica a capacidade dos países no enfrentamento dos riscos biológicos graves. $O$ artigo examina os dados da pandemia de 50 países para avaliar o poder preditivo do ISSG. A ausência da vacinação determinou difusão da COVID-19 no primeiro ciclo da pandemia em 2020. Os indicadores dos países são correlacionados e demonstrados por estatística descritiva. A metodologia de aglomeração por clusters agrupa os países segundo a similaridade da composição etária. A principal restrição que pode ser atribuida ao ISSG diz respeito ao privilegiamento das variáveis biomédicas para a mensuração da capacidade institucional. $O$ artigo evidencia que, paradoxalmente, o primeiro ciclo da pandemia teve um impacto significativo nos países teoricamente mais preparados, segundo o ISSG, para controlar a disseminação de doenças e oferecer mais acesso à assistência à saúde. O artigo assinala que durante o primeiro ciclo da pandemia, o setor saúde dependeu da cooperação dos governos na adoção do distanciamento social. $O$ ISSG não considerou o papel das lideranças politicas que desafiam o risco sanitário severo por veto às medidas de distanciamento social.

Palavras-chave Índice de Segurança Sanitária Global, COVID-19, Pandemia, Capacidade institucional, Análise comparada 


\section{Introdução}

Este texto analisa os efeitos da primeira onda da pandemia da COVID-19 em 50 Estados nacionais à luz do Índice da Segurança Sanitária Global - ISSG (The Global Health Security Index - GHSI) da Johns Hopkins University (JHU) ${ }^{1}$. Poucos estudos utilizaram o ISSG para descrever comparativamente as condições de segurança sanitária dos países durante os primeiros meses da pandemia ${ }^{2}$. Esta escassez foi surpreendente porque o ISSG agrega extensa documentação e dados quantitativos, de acesso aberto, que permitem a avaliação da capacidade institucional do setor saúde face aos riscos sanitários catástroficos ${ }^{3}$. Não resta dúvida que a compreensão da validade propositiva do ISSG tornou-se particularmente instigante diante da insegurança coletiva associada à disseminação em escala global do SARS-CoV-2 em 2020.

O ISSG apresenta um rol de indicadores complexos e úteis sobre a capacidade institucional dos países no enfrentamento dos riscos biológicos graves que causam danos em escala global, inviabilizam o desenvolvimento social e disseminam doenças letais. O ISSG é composto por seis variáveis: prevenção da emergência de epidemias; agilidade na detecção e informação sobre epidemias com alto potencial de risco; rapidez na resposta para mitigar a disseminação da epidemia; acessibilidade do setor saúde para assistir a população; comprometimento do país com as normativas de saúde internacionais, inclusive em termos financeiros e condição geral de risco ambiental e biológico do país ${ }^{1}$. O maior valor agregado do ISSG foi atribuído aos Estados Unidos (83,5 pontos). É importante destacar que predominam no ISSG as variáveis biomédicas da vigilância epidemiológica. Com a criação do ISSG, Johns Hopkins University defendeu enfaticamente a inclusão do tema dos riscos biológicos na agenda dos governos nacionais por força das interconexões mundiais que favorecem a criação e difusão de novos patógenos ${ }^{1}$.

Diante do evento de risco biológico extraordinário em curso, cabe considerar se a capacidade institucional do setor saúde medida pelo ISSG fez diferença na mitigação dos danos esperados pela disseminação do SARS-CoV-2. Não resta dúvida que a atual pandemia tem imposto ao ISSG monumental teste de estresse em função da escala dos casos e mortes associada ao novo coronavírus. Para Dalglish ${ }^{4}$, a saúde global nunca será a mesma após a COVID-19. Com base na amostra dos países da Organização para Cooperação e
Desenvolvimento Econômico (OCDE), Abbey et al. ${ }^{5}$ afirmam, por exemplo, que raros sistemas de saúde foram efetivos na resposta ao SARS-CoV-2 nos primeiros meses da pandemia. A baixa efetividade dos países da OCDE na resposta à COVID-19 comprovaria que o ISSG falhou na previsão dos países mais preparados para a emergência sanitária. Para os autores, a discrepância entre a classificação do ISSG e a resposta dos países da OECD à pandemia da COVID-19 comprovaria que ISSG subestimou o nível de preparo de muitos países e superestimou de outros. Os autores demandam inclusive que o painel de indicadores do ISSG seja reavaliado, incluindo especialmente a dimensão da "liderança política nacional" por força dos exemplos de sucesso da Nova Zelândia e da Coreia do Sul no início da pandemia ${ }^{5}$.

A conclusão dos autores deve ser vista com muita atenção porque ratifica a percepção de que as epidemias causadas pelos novos coronavírus (SARS-CoV-1, MERS e SARS-CoV-2) interromperam a estável relação da sociedade contemporânea com os eventos sanitários severos, alterando o padrão estabelecido pela pandemia da gripe espanhola de 1918-1919.

Nesses termos, é razoável esperar que os parâmetros dominantemente biológicos de qualificação das nações utilizados pela Johns Hopkins University tenham sido estressados em função da indisponibilidade de tecnologias farmacológicas (antiviral específico e vacina) para o tratamento da COVID-19 e controle dos SARS-CoV-2 ao longo do primeiro ciclo pandemia (março-dezembro de 2020).

Cabe destacar que, até o começo deste século, os Estados nacionais reduziram de modo contínuo a morbidade e a mortalidade por meio da centralização burocrática e especialização profissional para a provisão de bens coletivos no setor saúde $^{6,7}$. Mckeown ${ }^{8}$ chama a atenção para a contribuição, neste processo bem-sucedido, das intervenções de natureza técnico-científica, como controle de vetores, da imunização e da introdução contínua de novos fármacos, que promoveram a redução da morbidade e da mortalidade por doenças infecciosas, favorecendo o notável aumento da longevidade da população mundial ${ }^{8}$.

Em função da presente falha da intervenção em escala coletiva da biomedicina nas sucessivas ondas da pandemia em 2020, os governos nacionais foram, de fato, convocados a impor o distanciamento social massivo combinado com testagem, rastreamento de contato e quarentena ${ }^{9-11}$. Cabe assinalar que as primeiras vacinas para a COVID-19, só foram disponibilizadas para pou- 
cos países no começo de dezembro de 2020, nove meses após a decretação do estado de pandemia pela Organização Mundial de Saúde (OMS) ${ }^{12}$ Antes da vacina, as medidas de distanciamento social reduziram a velocidade da disseminação do SARS-CoV-2 em países asiáticos no começo da pandemia em $2020^{13}$. Portanto, ao longo de 2020, poucos países puderam reivindicar sucesso sustentável na contenção do SARS-Cov-2 ao adotar o distanciamento social massivo (lockdown) defendido pelos especialistas ${ }^{14}$.

$\mathrm{Na}$ realidade, o primeiro ciclo da pandemia, ao longo de 2020, caracterizado pela indisponibilidade das vacinas, exacerbou as preocupações com os grupos vulneráveis ${ }^{15}$, especialmente pela extrema letalidade da COVID-19 na população de idosos ${ }^{16,17}$ e do risco elevado de mortes da população obesa ${ }^{18}$.

\section{Material e métodos}

Para testar a consistência do ISSG como indicador da capacidade de resposta dos sistemas nacionais de saúde a eventos biológicos severos, este artigo examina os dados dos 50 países mais populosos ( $87 \%$ da população mundial em 2019). O artigo mediu o coeficiente de correlação $(r)$ do ISSG com a incidência acumulada e a mortalidade acumulada por 100 mil habitantes, a letalidade por 1.000 casos, a taxa de testagem e a sobremortalidade atribuídas à COVID-19 nos nove primeiros meses da pandemia.

O cálculo da sobremortalidade acumulada não período deriva da equação $\frac{\mathrm{dy}}{\mathrm{dx}}$,

onde $d y$ é a participação percentual do país no total global de óbitos atribuídos à COVID-19 e $d x$ é a participação percentual da população do país na população global em 2019. O resultado da operação maior que de 1 indica a condição de sobremortalidade do país.

O ISSG é também correlacionado as condições econômicas, demográficas e de oferta de serviços de saúde como renda per capita, proporção de pessoas com igual ou mais de 65 anos, proporção de obesos, idade média da população e disponibilidade de leitos.

A descrição por meio do coeficiente de correlação $r$ de variáveis quantitativas propõe identificar o grau de dependência especialmente dos indicadores de incidência e de mortalidade acumuladas por 100 mil habitantes em 2020 a variáveis estruturais na amostra dos países mais populosos. O indicativo de que há ou não correlação entre as variáveis selecionadas é o coeficiente de correlação, que assume valores entre -1 a +1 . Quanto maior o valor do coeficiente de correlação, independente de assumir valor positivo ou negativo, maior o grau de associação linear entre as variáveis ${ }^{19}$.

O artigo também utiliza a classificação dos 50 casos nacionais nos estratos de baixa, média e alta capacidade institucional do setor saúde segundo a Johns Hopkins ${ }^{1}$.

A amostra de 50 países se diferencia das amostras por conveniência dos estudos de avaliativos da efetividade explicativa do ISSG pela diversidade regional e alta assimetria nas condições de riqueza e oferta de leitos hospitalares.

Para medir a variabilidade dos casos selecionados, os indicadores são também descritos pela distribuição mediana e pelo coeficiente de variação. O coeficiente de variação mede a variabilidade relativa de uma distribuição e expressa o percentual do desvio padrão em relação à média ${ }^{20}$.

A metodologia de aglomeração por clusters ${ }^{21}$ é utilizada para agrupar os países segundo a similaridade da composição etária (pessoas com >65 anos e idade média da população).

As informações sobre o primeiro ciclo da pandemia da COVID-19 contemplam o período de $1^{\circ}$ de março de 2020 a 30 de novembro de 2020. Os dados analisados pela versão do SPSS 25.

As fontes dos dados são a publicação da Johns Hopkins University ${ }^{1}$, Banco Mundial ${ }^{22}$ e o sítio OurWorld in Data ${ }^{23}$. Os dados sobre a prevalência da obesidade na população adulta (índice de massa corporal igual ou superior a 30) são da Organização Mundial de Saúde ${ }^{24,25}$.

\section{Resultados}

O Quadro 1 mostra que apenas 6 países da amostra $(12 \%)$ foram classificados pelo escore global do ISSG como altamente capacitados no campo biomédico para oferecer resposta rápida a eventos sanitários severos: Canadá, França, Coreia do Sul, Tailândia, Estados Unidos e Reino Unido. No polo aposto, 10 países de grande porte (20\%) foram colocados na condição de baixa capacidade de responder com presteza a eventos epidemiológicos de alto risco, a maioria no continente africano. Os demais 34 países da amostra (68\%) teriam média capacidade de oferecer respostas com presteza às emergências associadas epidemias e pandemias. Chama a atenção no Quadro 1 que apenas um país de economia secundária (Tai- 
lândia) tenha sido classificado na condição de alto ISSG, como assinalam Abbey et al. ${ }^{5}$. Como mostra o Gráfico 1, a posição dos países no ISSG está positivamente correlacionada à riqueza dos países medida pelo PIB per capita.

A Tabela 1 mostra a incidência e os óbitos acumulados por 100 mil habitantes entre $1^{\circ}$ de março e 30 de novembro de 2020. Os coeficientes de variação dos indicadores da incidência acumulada e dos casos são elevadíssimos (respectivamente, $122 \%$ e $141 \%$ ), indicando que a disse- minação da COVID-19 apresentava um padrão completamente assimétrico na amostra analisadas dos 50 maiores países.

Contudo, a Tabela 2 mostra que os países com maiores índices de segurança sanitária são os que detêm também de maior proporção de idosos e idade média elevada. Os valores altos do ISSG estão também correlacionados às nações com grande quantidade de casos, óbitos, sobremortalidade pela COVID-19 e alta prevalência de população adulta obesa.

Quadro 1. Distribuição 50 Maiores dos Países Segundo o ISSG em novembro de 2019 (n=50).

\begin{tabular}{|l|l|c|}
\hline \multicolumn{1}{|c|}{ Condição no ISSG } & \multicolumn{1}{|c|}{ Países } & Média do ISSG \\
\hline Alta capacidade (n=6) & $\begin{array}{l}\text { Canadá, Coreia do Sul, Estados Unidos, França, Reino Unido e } \\
\text { Tailândia }\end{array}$ & 25,8 \\
\hline $\begin{array}{l}\text { Média Capacidade } \\
(\mathrm{n}=34)\end{array}$ & $\begin{array}{l}\text { Alemanha, Argentina, Arábia Saudita, Bangladesh, Brasil, } \\
\text { Colômbia, Egito, Filipinas, Gana, Índia, Indonésia, Irã, Itália, } \\
\text { Japão, Quênia, Malásia, México, Marrocos, Myanmar, Nepal, } \\
\text { Nigéria, Paquistão, Peru, Polônia e Rússia }\end{array}$ & 46,7 \\
\hline $\begin{array}{l}\text { Baixa Capacidade } \\
(\mathrm{n}=10)\end{array}$ & $\begin{array}{l}\text { Angola, Afeganistão, Argélia, Congo (República Democrática), } \\
\text { Etiópia, Iraque, Moçambique, Sudão, Iêmen e Venezuela }\end{array}$ & 74,7 \\
\hline
\end{tabular}

Fonte: Johns Hopkins, 2019.

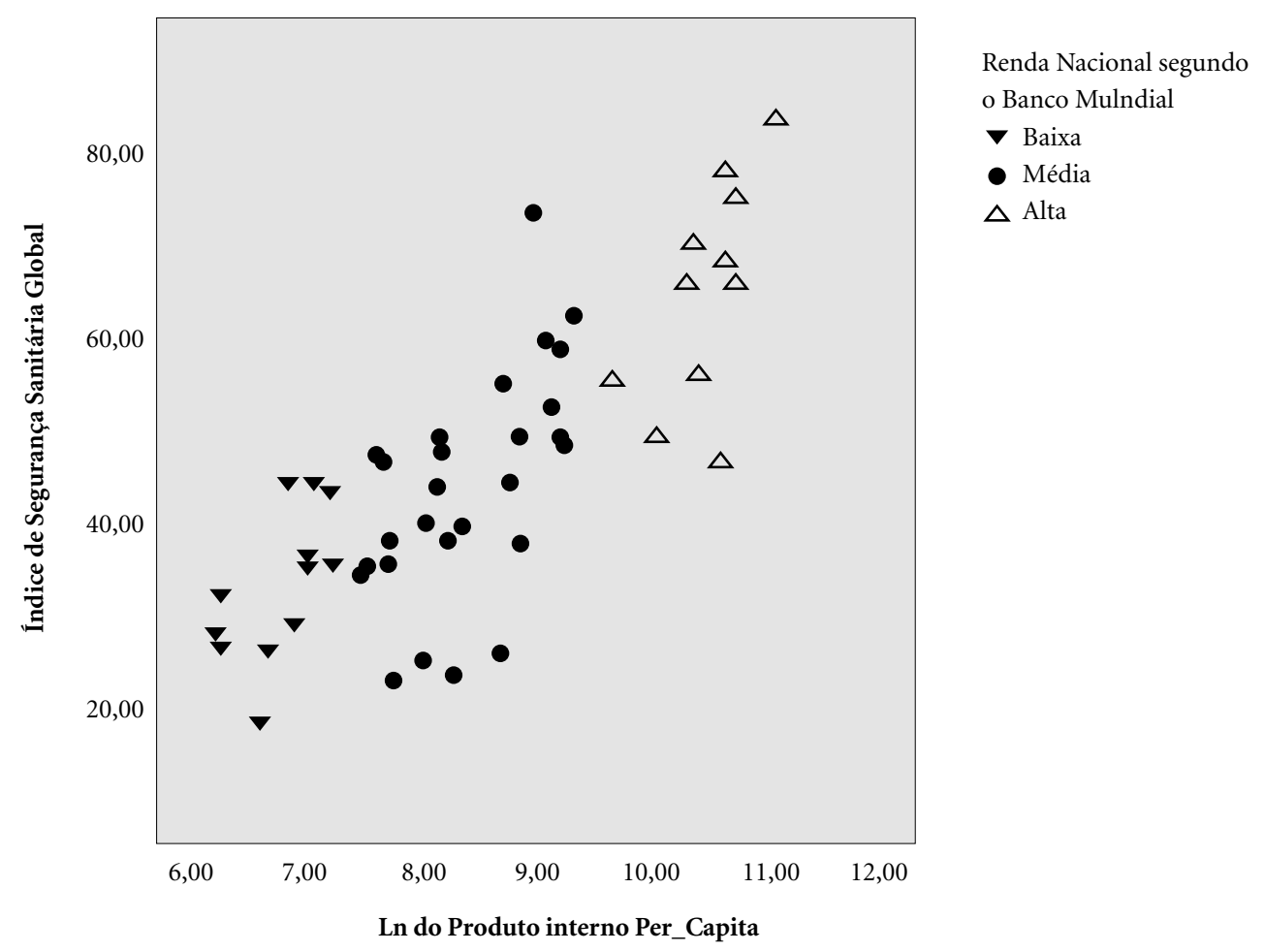

Gráfico 1. Distribuição do ISSG segundo o PIB per capita em 2019. 
Tabela 1. Distribuição da Incidência Acumulada e Mortes Acumuladas por 100 mil habitantes, atribuídas à COVID-19 entre $1^{\circ}$ de março e 30 de novembro de 2020, nos 50 países mais populosos do mundo.

\begin{tabular}{|c|c|c|}
\hline Países & $\begin{array}{c}\text { Incidência } \\
\text { Acumulada }\end{array}$ & $\begin{array}{c}\text { Mortalidade } \\
\text { Acumulada }\end{array}$ \\
\hline China & 6,40 & 0,33 \\
\hline Índia & 652,50 & 9,58 \\
\hline Estados Unidos & 3540,10 & 76,30 \\
\hline Indonésia & 176,80 & 5,70 \\
\hline Paquistão & 166,90 & 3,42 \\
\hline Brasil & 2814,20 & 79,07 \\
\hline Nigéria & 31,90 & 0,57 \\
\hline Bangladesh & 267,80 & 3,83 \\
\hline Rússia & 1397,80 & 23,88 \\
\hline México & 790,70 & 77,64 \\
\hline Japão & 99,00 & 1,54 \\
\hline Etiópia & 90,80 & 1,40 \\
\hline Filipinas & 377,30 & 7,30 \\
\hline Egito & 109,40 & 6,36 \\
\hline Vietnã & 1,30 & 0,04 \\
\hline RD Congo & 13,40 & 0,36 \\
\hline Turquia & 510,00 & 14,16 \\
\hline Irã & 954,70 & 51,13 \\
\hline Alemanha & 1049,80 & 16,27 \\
\hline Tailândia & 5,60 & 0,09 \\
\hline Reino Unido & 2140,70 & 79,21 \\
\hline França & 3192,50 & 72,20 \\
\hline Itália & 2146,20 & 79,17 \\
\hline Tanzânia & 85,20 & 0,03 \\
\hline África do Sul & 1280,90 & 34,85 \\
\hline Myanmar & 137,60 & 3,08 \\
\hline
\end{tabular}

O ISSG está também correlacionado aos países que implantaram testagem em massa como estratégia de vigilância epidemiológica e dispõe de maior oferta de leito em hospital. Ainda assim, a densidade na oferta de leitos não está correlacionada à menor incidência acumulada de óbitos.

A Tabela 2 mostra, portanto, que os países com ISSG mais elevados não apresentam letalidade significativamente diferente dos demais países, apesar dos seus sistemas nacionais de saúde terem sofrido monumental pressão de demanda por atendimento em 2020. A estrutura de oferta de leitos fez diferença apenas na mitigação da letalidade do SARS-CoV-2 nos países classificados com alto ISSG em comparação com os países pobres de baixa com baixa capacidade sanitária.
Tabela 1. Distribuição da Incidência Acumulada e Mortes Acumuladas por 100 mil habitantes, atribuídas à COVID-19 entre $1^{\circ}$ de março e 30 de novembro de 2020, nos 50 países mais populosos do mundo.

\begin{tabular}{|c|c|c|}
\hline Países & $\begin{array}{l}\text { Incidência } \\
\text { Acumulada }\end{array}$ & $\begin{array}{l}\text { Mortalidade } \\
\text { Acumulada }\end{array}$ \\
\hline Quênia & 137,80 & 2,47 \\
\hline Coreia do Sul & 58,50 & 0,98 \\
\hline Colômbia & 2408,40 & 68,32 \\
\hline Espanha & 3329,50 & 90,45 \\
\hline Uganda & 37,50 & 0,35 \\
\hline Argentina & 2985,70 & 8,03 \\
\hline Argélia & 163,40 & 5,07 \\
\hline Sudão & 34,30 & 2,68 \\
\hline Ucrânia & 1334,20 & 23,71 \\
\hline Iraque & 1315,70 & 29,42 \\
\hline Afeganistão & 113,30 & 4,24 \\
\hline Polônia & 2105,30 & 31,94 \\
\hline Canadá & 836,60 & 29,85 \\
\hline Marrocos & 844,10 & 13,79 \\
\hline Arábia Saudita & 1018,30 & 16,46 \\
\hline Uzbequistão & 212,30 & 1,80 \\
\hline Peru & 2863,00 & 107,50 \\
\hline Angola & 42,30 & 1,01 \\
\hline Malásia & 159,70 & 5,70 \\
\hline Moçambique & 47,10 & 0,38 \\
\hline Gana & 162,90 & 1,04 \\
\hline Iêmen & 7,00 & 2,04 \\
\hline Nepal & 738,00 & 4,38 \\
\hline Venezuela & 347,00 & 3,04 \\
\hline Média & 867 & 22 \\
\hline $\begin{array}{l}\text { Coeficiente de } \\
\text { variação }\end{array}$ & 122 & 141 \\
\hline
\end{tabular}

A Tabela 3 mostra que dois países com alto ISSG não implantaram a testagem em massa para orientar as decisões governamentais em relação ao distanciamento social (França e Tailândia). De modo geral, a testagem como ferramenta de vigilância epidemiológica da COVID-19 não foi implantada em 19 (38\%) dos países de grande porte, indicando que as informações sobre o número de casos e mesmo óbitos na amostra podem ser de baixa confiabilidade para muitos países. Os países de baixo ISSG mostraram extraordinária falta de comprometimento com a implantação da testagem em massa - apenas $2 \mathrm{em} 10$ o fizeram em 2020.

Até novembro de 2020, estavam também em condição de déficit na informação epidemiológica, pela falta de adoção da testagem massiva, ape- 
Tabela 2. Correlação do ISSG, PIB per capita, idade média da população, proporção de idosos, realização de teste, dummy para sobremortalidade, incidência, casos acumulados de COVID-19 e leitos por mil habitantes $(n=50)$.

\begin{tabular}{|c|c|c|c|c|c|c|c|c|c|c|}
\hline & ISSG & $\begin{array}{l}\text { PIB per } \\
\text { capita em } \\
2019 \text { (U\$) }\end{array}$ & $\begin{array}{l}\text { Obesidade } \\
\text { em } 2016\end{array}$ & $\begin{array}{c}\text { Proporção } \\
\text { da pop } \\
>65 \text { anos }\end{array}$ & $\begin{array}{c}\text { Testes por } \\
100 \text { mil } \\
\text { hab. }\end{array}$ & $\begin{array}{l}\text { Sobre- } \\
\text { morta- } \\
\text { lidade }\end{array}$ & $\begin{array}{l}\text { Casos } \\
\text { por } 100 \\
\text { mil hab. }\end{array}$ & $\begin{array}{l}\text { Mortes } \\
\text { por } 100 \\
\text { mil hab. }\end{array}$ & Leitos & Fatalidade \\
\hline ISSG & 1 & & & & & & & & & \\
\hline $\begin{array}{l}\text { PIB per capita em } \\
2019 \text { (U\$) }\end{array}$ & $0,763^{*}$ & 1 & & & & & & & & \\
\hline Obesidade em 2016 & $0,401^{*}$ & $0,463^{*}$ & 1 & & & & & & & \\
\hline $\begin{array}{l}\text { Proporção da pop } \\
>65 \text { anos em } 2019\end{array}$ & $0,653^{*}$ & $0,784^{*}$ & $0,386^{*}$ & 1 & & & & & & \\
\hline $\begin{array}{l}\text { Testes por mil } \\
\text { habitantes }\end{array}$ & $0,573^{*}$ & $0,664^{*}$ & $0,587^{\star}$ & $0,556^{\star}$ & 1 & & & & & \\
\hline $\begin{array}{l}\text { Dummy para } \\
\text { sobremortalidade } \\
\text { pela COVID-19 }\end{array}$ & $0,469^{*}$ & $0,486^{*}$ & $0,782^{\star}$ & $0,479^{*}$ & $0,471^{\star}$ & 1 & & & & \\
\hline $\begin{array}{l}\text { Casos por } 100 \mathrm{mil} \\
\text { habitantes }\end{array}$ & $0,553^{*}$ & $0,526^{*}$ & $0,632^{*}$ & $0,519^{*}$ & $0,525^{\star}$ & $0,723^{*}$ & 1 & & & \\
\hline $\begin{array}{l}\text { Mortes por } 100 \mathrm{mil} \\
\text { habitantes }\end{array}$ & $0,493^{*}$ & $0,483^{*}$ & $0,579^{*}$ & $0,446^{*}$ & $0,488^{\star}$ & $0,781^{\star}$ & $0,846^{*}$ & 1 & & \\
\hline $\begin{array}{l}\text { Leitos por } 100 \\
\text { habitantes }\end{array}$ & $0,408^{\star}$ & $0,505^{\star}$ & 0,192 & $0,732^{*}$ & 0,269 & 0,245 & 0,2015 & 0,70 & 1 & \\
\hline Fatalidade & $-0,215$ & $-0,072$ & 0,068 & $-0,120$ & $-0,083$ & $-0,010$ & $-0,106$ & 0,068 & $-0,145$ & 1 \\
\hline
\end{tabular}

*Nível de significância de 0,001.

Fonte: Johns Hopkins, Ourworld in Data.

Tabela 3. Implementação de Testagem em Massa nos Países segundo o ISSG nos Países Populosos (>acima de 28 milhões de habitantes $)(n=50)$.

\begin{tabular}{lccc}
\hline $\begin{array}{c}\text { Posição } \\
\text { na Escala } \\
\text { do ISSG }\end{array}$ & $\begin{array}{c}\text { Não Faz } \\
\text { Testagem em } \\
\text { Massa (A) }\end{array}$ & Total (B) & $\begin{array}{c}\text { (Razão } \\
\text { A/B) }{ }^{*} \mathbf{1 0 0}\end{array}$ \\
\hline Baixa & 8 & 10 & $80 \%$ \\
Média & 9 & 34 & $26 \%$ \\
Alta & 2 & 6 & $33 \%$ \\
Total & 19 & 50 & $38 \%$ \\
\hline
\end{tabular}

Fonte: Johns Hopkins, Ourworld in Data. sar da sobremortalidade por COVID-19, o Brasil e França. Os demais grandes países com déficit informacional pela não aplicação da testagem massiva, mas com a mortalidade informada abaixo do esperado em relação proporção da sua população na amostra eram Afeganistão, Argélia, Angola, Argentina, China, República Democrática do Congo, Egito, Quênia, Moçambique, Myanmar, Sudão, Tanzânia, Tailândia, Uzbequistão, Venezuela, Vietnam e Iêmen.
Por fim, os dados da Tabela 4 mostram que a estrutura demográfica foi um fator de risco para a mortalidade associada à disseminação do novo coronavírus ao longo de 2020, como indicou a literatura. Os países com alta proporção de idosos, com exceção do Japão, tiveram uma taxa de mortalidade 24 vezes maior do que os países com estrutura demográfica jovem. Os dados da Tabela 4 ressaltam também a lenta transição demográfica nos países da África na amostra: a proporção de população idosa é residual no cluster de países agregados como de "população muito jovem" (3\%). O predomínio das nações do continente africano neste cluster é notável.

\section{Conclusão}

Este artigo problematiza criticamente Índice da Segurança Sanitária Global (ISSG) da Johns Hopkins University como um indicador efetivo para estimar a capacidade de resposta dos países durante o primeiro ciclo da pandemia da COVID-19 em 2020 face à indisponibilidade de vacinas. A principal restrição que pode ser atribuída ao ISSG diz respeito ao privilegiamento das variáveis biomédicas para a mensuração da capacida- 
Tabela 4. Mediana da proporção de pessoas com $>65$ anos, produto interno bruto per capita e mortes por 100 mil habitantes pela COVID-19, segundo os clusters dos países pela estrutura demográfica.

\begin{tabular}{|c|c|c|c|c|}
\hline Posição na Estrutura Demográfica & $\begin{array}{c}\text { Proporção } \\
\text { da População } \\
\text { com }>65 \text { anos }\end{array}$ & $\begin{array}{l}\text { PIB per capita } \\
\text { por PPC (US\$) } \\
-2019\end{array}$ & $\begin{array}{c}\text { Leitos } \\
\text { por } 1000 \\
\text { habitantes }\end{array}$ & $\begin{array}{l}\text { Mortes pela } \\
\text { COVID-19 por } \\
\text { 100K habitantes }\end{array}$ \\
\hline Cluster População Muito Jovem $(\mathrm{n}=14)$ & 3 & 1010 & 0,65 & 1,2 \\
\hline Cluster População Jovem ( $\mathrm{n}=22)$ & 6,5 & 4050 & 1,6 & 7,7 \\
\hline Cluster População Mais Velha $(\mathrm{n}=13)$ & 18 & 31850 & 4,3 & 29,9 \\
\hline Japão $(\mathrm{n}=1)$ & 28 & 40260 & 13,1 & 1,5 \\
\hline Total $(n=50)$ & 6,5 & 3085 & 1,6 & 5,7 \\
\hline
\end{tabular}

Cluster População Muito Jovem: Angola, Afeganistão, Congo (República Democrática), Etiópia, Gana, Iraque, Paquistão, Quênia, Moçambique, Nigéria, Sudão, Tanzânia, Uganda e Iêmen. Cluster População Jovem: Argentina, Argélia, Bangladesh, Brasil, Colômbia, Egito, Îndia, Indonésia, Irã, Peru, Malásia, México, Marrocos, Mianmar, Nepal, Filipinas, Arábia Saudita, África do Sul, Turquia, Uzbequistão, Venezuela e Vietnã. Cluster População Mais Velha: Alemanha, Canadá, China, Coreia do Sul, França, Espanha, Itália, Polônia, Rússia, Tailândia, Ucrânia, Reino Unido e Estados Unidos.

Fonte: Ourworld in Data.

de institucional das nações. Por força dessa configuração, o artigo demonstra que os países com o ISSG mais elevado foram duramente desafiados pela ausência de ferramentas farmacológicas para controlar a disseminação do SARS-CoV-2 e a mortalidade causadas pela COVID-19 em 2020.

A falta de ferramentas farmacológicas deixou o setor saúde desses países fortemente dependente da cooperação da sociedade e governos para implantar o distanciamento social. O ISSG não contemplou a hipótese de que as lideranças políticas pudessem desafiar o risco sanitário severo, negando-se a implantar as medidas de distanciamento social. Este artigo chama a atenção que, em muitos países de grande porte e ricos, a prescrição do distanciamento social para controlar a disseminação do SARS-CoV-2 foi objeto de veto político do governo central ao longo de 2020 .

Nesse contexto, a questão específica da orientação da liderança governamental em relação à ciência ${ }^{10}$ fragilizou a tentativa da JHU em construir um índice de capacidade institucional do setor saúde em escala global. Muitos países fracassaram na construção do consenso sobre a severidade da COVID-19 em razão do negacionismo militante dos governos centrais. Os exemplos de negacionismo dos governos centrais de Brasil $^{26,27}$, Estados Unidos ${ }^{28}$ e México ${ }^{29}$ são emblemáticos. Os Estados Unidos responderam de modo errático ao ciclo da pandemia de 2020 em função do negacionismo científico do governo Trump ${ }^{30}$, apesar do primeiro lugar na classificação do ISSG. Não resta dúvida que o ISSG pode aprimorar sua métrica no sentido de dar mais destaque às variáveis políticas e institucionais das nações.
Já é consensual o reconhecimento de que a atuação do governo central na implantação com presteza do distanciamento social e na disseminação da informação científica fez a diferença em países específicos, permitindo que o setor saúde conquistasse o apoio da maioria da sociedade durante o primeiro ciclo da pandemia de $2020^{31}$.

A baixa incidência de casos e dos óbitos em países com baixo ISSG, identificada no artigo, também pode, assim, ser explicada por escolhas da sociedade, como demonstrada na experiência de outras epidemias igualmente severas neste século $^{32}$.

Como assinala Dalglish ${ }^{4}$, o aprendizado social de controle dos coronavírus, presente em países asiáticos, também foi mimetizado por nações com baixo ISSG. Não resta dúvida que a mobilização cívica e a indução governamental dessas sociedades podem ter possibilitado a mitigação da ameaça biológica catastrófica que foi a disseminação global do SARS-CoV-2 em 2020.

Em resumo, o maior paradoxo evidenciado neste artigo é que a pandemia declarada pela OMS em 11 de março de $2020^{25}$ teve um impacto devastador nos países teoricamente mais preparados, segundo o ISSG, para controlar a disseminação de doenças e oferecer mais acesso à assistência à saúde em 2020. A análise com base na amostra dos grandes países evidencia, assim, o fracasso dos sistemas nacionais de saúde da maioria das nações ricas e emergentes na proteção das populações contra o SARS-CoV-2. A sobremortalidade produzida pela COVID-19 esteve diretamente correlacionada aos sistemas de saúde dos países de alta renda que receberam a 
classificação de elevada capacidade institucional para lidar com emergência sanitária no ISSG. Ao contrário do esperado, estes países não demonstraram capacidade de lidar com a emergência do SARS-CoV-2 em 2020.

Algumas características estruturais dos países de alta renda podem explicar este fracasso. Este artigo identifica a influência da estrutura demográfica e da distribuição da obesidade na surpreendente na concentração da sobremortalidade nos países que foram classificados com escores elevados do ISSG em comparação aos países com estrutura populacional mais jovem. A falta de ferramentas farmacológicas também contribuiu para a relativa falha da maioria das nações ricas em oferecer assistência aos idosos e pessoas obesas, mesmo nas nações com sistema de saúde com alta densidade de oferta de leitos em hospitais.
Por outro lado, o bônus demográfico (população muito jovem) pode ter favorecido o bom desempenho das nações com baixo ISSG quando confrontadas pelo SARS-Cov-2 ao longo do primeiro ciclo da pandemia de 2020. Contudo, é necessário também chamar à atenção para a possibilidade de que os países com baixo ISSG tenham sido beneficiados, nas informações divulgadas pelo sítio Ourworld in Data, pela subnotificação sistêmica derivada da incapacidade governamental de detectar e reportar doenças e causas de morte. Cabe ressaltar, como mostrado no artigo, que quase $80 \%$ dos países mais populosos com baixo ISSG e taxas residuais de incidência da COVID-19, não realizaram a testagem em massa para o rastreamento dos casos e causas de óbitos, colocando em dúvida a validade e a confiabilidade dos dados divulgados ao longo do ciclo de 2020.

\section{Colaboradores}

NR Costa participou da concepção, análise e interpretação dos dados, redação do artigo e aprovação da versão a ser publicada. PRF Silva, MJ Lago e A Jatobá participaram do delineamento e interpretação dos dados para o trabalho, revisão crítica do conteúdo intelectual e aprovação da versão a ser publicada. 


\section{Referências}

1. Johns Hopkins University (JHU). The Global Health Security Index. Baltimore: JHU; 2019.

2. Boyd MJ, Wilson N, Nelson C. Validation analysis of Global Health Security Index (GHSI) scores 2019 BMJ Global Health 2020; 5:e003276.

3. Ravi SJ, Warmbrod KL, Mullen L, Meyer D, Cameron E, Bell J, Bapat P, Paterra M, Machalaba C, Nath I, Gostin LO, James W, George D, Nikkari S, Gozzer E, Tomori O, Makumbi I, Nuzzo JB. The value proposition of the Global Health Security Index. BMJ Global Health 2020; 5:e003648.

4. Dalglish SL. COVID-19 gives the lie to global health expertise. Lancet 2020; 395(11):1189.

5. Abbey EJ, Khalifa BAA, Oduwole MO, Ayeh SK, Nudotor RD, Salia EL, Lasisi O, Bennett S, Yusuf HE, Agwu AL, Karakousis PC. The Global Health Security Index is not predictive of coronavirus pandemic responses among Organization for Economic Cooperation and Development Countries. PLoS One 2020; 15(10):e0239398.

6. de Swaan A. In Care of the State: Health, Education and Welfare in Europe and USA in the Modern Era. Oxford: Oxford University Press; 1988.

7. Porter D. Health, Civilization and the State - a history of public health from ancient to modern times. London: Routledge; 1999.

8. Mckeown T. The Role of Medicine. Dream, Mirage, or Nemesis. New Jersey: Princeton University Press; 1979.

9. Lipsitch M. We know enough now to act decisively against COVID-19. Social distancing is a good place to start [Internet]. Stat; 2020 [acessado 2020 nov 20]. Disponível em: https://www.statnews. com/2020/03/18/we-know-enough-now-to-act-decisively-against-covid-19/.

10. Kavanagh MM, Singh R. Democracy, Capacity, and Coercion in Pandemic Response: COVID-19 in Comparative Political Perspective. J Health Polit Policy Law 2020; 45(6):997-1012.

11. Ferguson NM, Laydon D, Nedjati-Gilani G, Imai N, Ainslie K, Baguelin M, Bhatia S, Boonyasiri A, Cucunubá Z, Cuomo-Dannenburg G, Dighe A, Dorigatti I, Fu H, Gaythorpe K, Green W, Hamlet A, Hinsley W, Okell LC, van Elsland S, Thompson H, Verity R, Volz E, Wang H, Wang Y, Walker PGT, Walters C, Winskill P, Whittaker C, Donnelly CA, Riley S, Ghani AC. The Impact of non-pharmaceutical interventions (NPIs) to reduce COVID-19 mortality and healthcare demand [Internet]. Imperial College London; 2020 [acessado 2020 nov 20]. Disponível em: https://www.imperial. ac.uk/media/imperial-college/medicine/sph/ide/ gida-fellowships/Imperial-College-COVID19-NPImodelling-16-03-2020.pdf.

12. The Guardian. Covid vaccine: UK woman becomes first in world to receive Pfizer jab [Internet]. 2020 [acessado $2020 \mathrm{dez} 10$ ]. Disponível em: https://www. theguardian.com/world/2020/dec/08/coventry-woman-90-first-patient-to-receive-covid-vaccine-innhs-campaign.
13. Ainslie KEC, Walters CE, Fu H, Bhatia S, Wang H, Xi X, Baguelin M, Bhatt S, Boonyasiri A, Boyd O, Cattarino L, Ciavarella C, Cucunuba Z, Cuomo-Dannenburg G, Dighe A, Dorigatti I, van Elsland SL, FitzJohn R, Gaythorpe K, Ghani AC, Green W, Hamlet A, Hinsley W, Imai N, Jorgensen D, Knock E, Laydon D, Nedjati-Gilani G, Okell LC, Siveroni I, Thompson HA, Unwin HJT, Verity R, Vollmer M, Walker PGT, Wang Y, Watson OJ, Whittaker C, Winskill P, Donnelly CA, Ferguson NM, Riley S. Evidence of Initial success for China exiting COVID-19 social distancing policy after achieving containment. Wellcome Open Res 2020; $5: 81$.

14. The Economist. Why Rich Countries Are So Vulnerable to COVID-19 [Internet]. 2020 [acessado 2020 nov 20]. Disponível em: https://www.economist.com/graphicdetail/2020/11/16/why-rich-countries-are-so-vulnerable-to-COVID-19.

15. Mustaffa N, Lee S-Y, Nawi SNM, Rahim MJC, Chee YC, Besari AM, Lee YY. COVID-19 in the elderly: A Malaysian perspective. J Glob Health 2020; 10(2):020370.

16. Ho FK, Petermann-Rocha F, Gray SR, Jani BD, Katikireddi SV, Niedzwiedz CL, Foster H, Hastie CE, Mackay DF, Gill JMR, O'Donnell C, Welsh P, Mair F, Sattar N, Celis-Morales CA, Pell JP. Is Older Age Associated with COVID-19mortality in the absence of other risk factors? General population cohort study of 470,034 participants. PLoS One 2020; 15(11):e0241824.

17. Hoffmann C, Wolf E. Older age groups and country -specific case fatality rates of COVID-19 in Europe, USA and Canada. Infection 2021; 49(1):111-116.

18. Busetto L, Bettini S, Fabris R, Serra R, Dal Pra C, Maffei P, Rossato M, Fioretto P, Vettor R. Obesity and COVID-19: An Italian Snapshot. Obesity 2020; 28(9):1600-1605.

19. Agresti A, Finlay B. Statistical Methods for the Social Sciences. New Jersey: Prentice Hall; 1997.

20. Daniel WW. Biostatistics. A Foundation for Analysis in the Health Sciences. New Jersey: John Wiley \& Sons, Inc.; 2009.

21. Everitt B. Cluster Analysis. London: Heinemann Educational Books; 1974.

22. The World Bank [Internet]. [acessado 2020 nov 12]. Disponível em: https://data.worldbank.org/.

23. Ourworld in Data [Internet]. [acessado 2020 nov 17]. Disponível em: https://ourworldindata.org/grapher/ total-covid-deaths-per-million?tab=chart\&country $=$ BRA.

24. World Health Organization (WHO). Global Health Observatory Indicator Views [Internet]. [acessado 2020 nov 12]. Disponível em: https://apps.who.int/ gho/data/node.imr.NCD_BMI_30C?lang=en.

25. World Health Organization (WHO). WHO announces COVID-19 outbreak a pandemic [Internet]. [acessado 2020 nov 14]. Disponível em: euro.who.int/en/ health-topics/health-emergencies/coronavirus-COVID-19/news/news/2020/3/who-announces-COVID19-outbreak-a-pandemic.

26. Lancet Editorial. COVID-19 in Brazil. "So What?" Lancet 2020; 395(10235):1461. 
27. Phillips D. Brazil: largest rise in COVID-19 deaths follows Bolsonaro 'worst is over' claim [Internet]. The Guardian; 2020 [acessado 2020 nov 14]. Disponível em: https://www.theguardian.com/world/2020/ may/06/brazil-coronavirus-deaths-covid-19-bolsonaro.

28. Gabbat A. Trump aides seek to discredit Fauci over coronavirus crisis as cases surge [Internet]. The Guardian; 2020 [acessado 2020 nov 14]. Disponível em: https:// www.theguardian.com/world/2020/jul/13/trump-administration-fauci-coronavirus-crisis.

29. Agren D. Mexican president Amlo says he will wear mask 'when there is no corruption [Internet]. The Guardian; 2020 [acessado 2020 nov 11]. Disponível em: https://www.theguardian.com/world/2020/ jul/31/mexico-president-amlo-mask-coronaviruscorruption.

30. Buranyi S. Attacked by Trump and Ignored by many of its Most Powerful Members, the World Health Organization is Facing a Major Crisis [Internet]. The Guardian; 2020 [acessado 2020 nov 11]. Disponível em: https://www.theguardian.com/news/2020/apr/10/ world-health-organization-who-v-coronavirus-why -it-cant-handle-pandemic.

31. Nuzzo JB, Bell JA, Cameron EE. Suboptimal US Response to COVID-19 Despite Robust Capabilities and Resources. JAMA 2020; 324(14):1391-1392.

32. Legido-Quigley H, Asgari N, Teo YY, Leung GM, Oshitani H, Fukuda K, Cook AR, Hsu LY, Shibuya K, Heymann D. Are high-performing health systems resilient against the COVID-19 epidemic? Lancet 2020; 395(10227):848-850.

Artigo apresentado em 20/11/2020

Aprovado em 31/05/2021

Versão final apresentada em 02/06/2021

Editores-chefes: Romeu Gomes, Antônio Augusto Moura da Silva 